\title{
On iterated functions with asymptotic conditions at a fixpoint
}

\author{
By Anders Lundberg
}

\section{Introduction}

It is a known fact that the problem of embedding a real function $f$ in a family $\left\{f_{\alpha}\right\}$ of iterates, where $\alpha$ denotes an arbitrary real number, has no unique solution. A chief problem in iteration theory is that of finding and describing suitable uniqueness conditions for the iterates. It appears that a natural way to obtain uniqueness is to require something about the behaviour of the functions $f_{\alpha}$ in a neighbourhood of a fixpoint. Many authors have dealt with such conditions, but in this connection we mention only Fort [3] and Michel [4]. Michel has given uniqueness conditions for three cases concerning the behaviour of $f$ at a fixpoint. For these cases some existence theorems are given by Szekeres [5]. In this paper we give some extensions of these theorems

In Sections 2 and 3 below, we state the basic concepts used here and some simple results concerning iteration in general. In Sections 4 and 5 we give the main theorems for extending the results by Michel and Szekeres. Finally, in Section 6, we quote Michel's uniqueness theorems and show a way to extend them.

\section{Some definitions}

For convenience we shall consider a rather special class of functions, but this fact makes no essential restriction of the results obtained (see [4], Section 5).

Some of the concepts and notations introduced below are in accordance with those given by Michel [4]. In the following we shall consider functions which are continuous and strictly increasing, and this property is called c.s.i.

Definition 2.1. A function $f$ belongs to the class, $S$ if, and only if,

(a) $f(x)$ is defined and c.s.i. for $x \geqslant 0$,

(b) $f(0)_{1}=0, f(\infty)=\infty$.

Definition 2.2. $B y S_{0}$ we mean the subclass of $S$ consisting of $e$, where $e(x)=x$ for every $x$, and the functions $f$ such that $f(x) \neq x$ for every $x>0$.

Definition 2.3. $A$ set $G=\left\{f_{\alpha}\right\} \subset S_{0},-\infty<\alpha<\infty$, is called a continuous iteration group if, and only if,

$$
f_{\alpha} f_{\beta}=f_{\beta} f_{\alpha}=f_{\alpha+\beta}
$$


A. LUNDBERG, Iterated functions with asymptotic conditions at a fixpoint

and

$$
\lim _{t \rightarrow \alpha} f_{t}(x)=f_{\alpha}(x)
$$

$f_{\alpha} f_{\beta}$ means the function obtained by composition, i.e. for every $x \geqslant 0$ we have $f_{\alpha} f_{\beta}(x)=f_{\alpha}\left(f_{\beta}(x)\right)$. From (1) it follows that $f_{0}=e$. If a given function $f \in S_{0}$ is embedded in $G$ and if $f_{1}=f$, we shall call $G=G(f)$ an iteration group of $f$. In this case $f_{1}, f_{2}, \ldots$ are the natural iterates of $f$, and $f_{-1}$ is the inverse of $f$.

In the following we shall always let $f$ and $g$ denote functions in $S_{0}$ different from $e$, and $F$ be a function in $S . F_{-1}$ shall always denote the inverse of $F$. The iteration groups below are always assumed to be continuous.

\section{Existence and general properties of iteration groups}

The existence of iteration groups is trivial. For instance, $\left\{f_{\alpha} \mid f_{\alpha}(x)=k^{\alpha} x\right\}$ is an iteration group if $k>0$.

Lemma 3.1. Let $f_{1}<e$ and $x>0$. Then $\lim _{n \rightarrow \infty} f_{n}(x)=0$, and $\lim _{n \rightarrow \infty} f_{-n}(x)=\infty$.

Proof. Both sequences are monotonic, and they must tend to a fixpoint of $f_{1}$.

Lemma 3.2. Let $f \neq e$ and let $\left\{f_{\alpha}\right\}$ be a continuous iteration group of $f$. For any fixed $x>0, f_{\alpha}(x)$, regarded as a function of $\alpha$, defines a mapping of $(-\infty$, $+\infty)$ onto $(0,+\infty)$ which is continuous and strictly monotone, increasing if $f>e$ and decreasing if $f<e$.

Proof. The strict monotonicity is proved in [2]. The other propositions follow from Lemma 3.1.

Lemma 3.3. Let $\alpha \neq 0$ be a given number, and let $g=f_{\alpha} \in G(f)$. Then the set $\left\{g_{\beta} \mid g_{\beta}=f_{\alpha \beta}\right\}$ equals the set $G(f)$ and is an iteration group of $g$.

The proof is obvious.

Lemma 3.4. If $\left\{f_{\alpha}\right\}$ is a given continuous iteration group and if $F \in S$, then $\left\{F f_{\alpha} F_{-1}\right\}$ is also a continuous iteration group. If $\left\{g_{\alpha}\right\}$ is an arbitrary continuous iteration group, and if $f_{1}$ and $g_{1}$ are both either $>e$ or $<e$, then there is an $F \in S$ such that $g_{\alpha}=F f_{\alpha} F_{-1}$.

Proof. The first statement is obvious. To prove the other statement, we let $a>0$ be a fixed number. By Lemma 3.2, for every $x>0$ there is one and only one number $\beta=\beta(x)$ such that $x=f_{\beta}(a)$; in particular, $\beta(a)=0$. If we define $F(0)=0$ and $F(x)=g_{\beta}(c)$, where $c=F(a)>0$ may be arbitrarily chosen, we obtain from Lemma 3.2 that $F \in S$. We have $F f_{\alpha}(x)=F f_{\alpha+\beta}(a)=g_{\alpha+\beta}(c)=g_{\alpha} F(x)$, which proves the statement.

If $f$ is a given function and $\left\{g_{\alpha}\right\}=G(g)$ an iteration group, where $f$ and $g$ are both either $>e$ or $<e$, then from Lemma 3.4 we obtain that the problem of embedding $f$ in an iteration group is equivalent to that of solving the equation $F f(x)=g F(x)$. In particular, if $g_{\alpha}(x)=k^{\alpha} x, 0<k \neq 1$, we get the Schröder equation

$$
F f(x)=k \cdot F(x)
$$


with multiplier $k$. It is a rather trivial fact that we can construct solutions of (3) if we do not require more than $F \in S$. For this construction, see e.g. Walker [7].

A solution $F \in S$ of (3) shall be called a Schröder function of $f$. If for an iteration group $\left\{f_{\alpha}\right\}, F f_{\alpha}(x)=k^{\alpha} \cdot F(x)$ for every $\alpha$, then we shall call $F$ a Schröder function of $\left\{f_{\alpha}\right\}$.

Lemma 3.5. If $F$ is a Schröder function of $\left\{f_{\alpha}\right\}$, then any other Schröder function of $\left\{f_{x}\right\}$ has the form $c \cdot F^{b}$, where $b$ and $c$ are constants $>0$.

Proof. Let $F_{1}$ and $F_{2}$ be two Schröder functions of $\left\{f_{\alpha}\right\}$ with multipliers $k_{1}$ and $k_{2}$ respectively, and suppose $k_{1}^{b}=k_{2}$. Then for every $x>0$ and $\alpha$ we have

$$
\frac{\left(F_{1} f_{\alpha}(x)\right)^{b}}{F_{2} f_{\alpha}(x)}=\frac{\left(F_{1}(x)\right)^{b}}{F_{2}(x)}
$$

Keeping $x$ fixed, let $\alpha$ range over the real numbers. The proposition then follows from Lemma 3.2.

Sometimes it is convenient to use the Abel equation,

$$
A f(x)=\mathbf{1}+A(x),
$$

obtained from (3) by putting $A(x)={ }_{k} \log F(x)$. As with Schröder functions, we also speak of Abel functions of $f$ and of $\left\{f_{\alpha}\right\}$. By means of a given Abel function of $f$, we can obtain every iteration group of $f$ as follows:

Lemma 3.6. Let $A$ be an Abel function of $f$ and put $B(x)=A(x)+p A(x)$, where $p$ is periodic with period 1 , and $p+e$ is c.s.i. Then $B$ also is an Abel function of $f,\left\{f_{\alpha} \mid f_{\alpha}(0)=0, f_{\alpha}(x)=B_{-1}(\alpha+B(x))\right.$ for $\left.x>0\right\}$ is an iteration group of $f$, and every $G(f)$ can be obtained in this way by suitable choice of $p$. $A$ and $B$ generate the same iteration group if, and only if, $p=$ const.

The proof is obvious and is therefore omitted. We may note that the main content of this lemma is already known; see e.g. Szekeres [5] or Töpfer [6].

\section{Differentiability at a fixpoint}

A function $f$, which has a right derivative $f^{\prime}(0)$ at zero, does not necessarily have an iteration group such that $f_{\alpha}^{\prime}(0)$ exists for every $\alpha$. We now consider a class of functions having iteration groups with this property.

Definition 4.1. A function $f \in S_{0}$ belongs to the class $K$ if and only if $f$ has $a$ continuous iteration group $G(f)$ such that $f_{\alpha}^{\prime}(0)$ exists for every $f_{\alpha} \in G(f)$, and for $\alpha \neq 0$,

$$
0<f_{\alpha}^{\prime}(0) \neq 1 \text {. }
$$

From Lemma 3.3 we immediately obtain that if every function of $G(f)$ satisfies (4), then $G(f) \subset K$. 
A. LUNDBERG, Iterated functions with asymptotic conditions at a fixpoint

We shall give some conditions for belonging to $K$, and for this we introduce the following notations, used throughout this section:

If a number $k$ is associated with a function $f$, we let this fact imply that $f^{\prime}(0)$ exists, that $k=f^{\prime}(0)$, and that $f^{\prime}(0)$ satisfies (4).

By $Q$ we mean the first quadrant, i.e. the set $\{(x, y) \mid x>0, y>0\}$. For $(x, y) \in Q$, we put

$$
\varphi_{n}(x, y)=\frac{f_{n}(x)}{f_{n}(y)} \quad(n=1,2,3, \ldots)
$$

If $f<e$, we put

$$
\varphi(x, y)=\lim _{n \rightarrow \infty} \varphi_{n}(x, y)
$$

for $(x, y) \in D_{\varphi}$, where $D_{\varphi}$ is the subset of $Q$ consisting of all points $(x, y)$ such that the limit (5) exists. Sometimes, when $y$ is kept fixed and we regard $\varphi$ as a function of $x$ only, we write $\varphi(x)$ instead of $\varphi(x, y)$, if nothing can be misunderstood. In this case, we let $\varphi_{-1}$ denote the inverse of $\varphi$.

The following theorem gives a necessary and sufficient condition that $f \in K$, and it also shows the known fact (see [4]) that the differentiability at a fixpoint makes the iteration group unique.

Theorem 4.2. Let $f$ be a function with $k<1$, and let $a>0$ be an arbitrary fixed number. If $f \in K$, then $\varphi(x, a)$ exists for every $x>0$ and is a c.s.i. function of $x$. There is only one continuous iteration group $G(f) \subset K$; and $\varphi$, together with $f_{\alpha}$, where $f_{\alpha} \in G(f)$, satisfies

$$
\varphi\left(f_{\alpha}(x), a\right)=k^{\alpha} \cdot \varphi(x, a)
$$

Conversely, if $\varphi(x, a)$ exists for every $x>0$ and is c.s.i., then $f \in K$.

A weaker variant of this theorem is proved by Szekeres [5].

Remarks: In the theorem we have assumed that $k<1$, which, of course, implies that $f<e$. This restriction is made in order to ensure that $f_{n}(x) \rightarrow 0$, when $n \rightarrow \infty$. But the theorem can be applied to functions $f>e$, if we use $f_{-1}$ instead of $f$. In fact, $f \in K$ if, and only if, $f_{-1} \in K$.

By definition, the condition that $f^{\prime}(0)$ exists and satisfies (4) is necessary for $f \in K$, but it is not sufficient. If $0<f^{\prime}(0)<1$, it may still happen that $\varphi(x)$ does not exist for every $x>0$. It may also happen that $\varphi$ exists but has jumps or constancy intervals. The latter is exemplified in Szekeres [5].

The condition of the above theorem is not easily applicable, because it does not clearly express the behaviour of $f$ in a neighbourhood of 0 . Therefore, we give some sufficient conditions involving simple properties of $f$ :

Theorem 4.3. Suppose that $k<1$. Let $d$ be a fixed number $>0$. Then each of the following three conditions implies that $f$ belongs to $K$ :

(a) $f^{\prime}(x)$ exists for $0<x<d$, and there is a number $\delta>0$ such that

$$
f^{\prime}(x)=k+0\left(x^{\delta}\right) \quad(x \rightarrow 0) ;
$$

\footnotetext{
1 This condition is due to Szekeres [5].
} 
(b) $f(x) / x$ is an increasing function of $x$ for $0<x<d$;

(c) $f$ is concave in $(0, d) .1$

Remark: Every convex function satisfies $(b)$. The corresponding condition, satisfied by concave functions, would be that $f(x) / x$ is a decreasing function of $x$. It is a surprising fact, however, that this condition is not sufficient for $f \in K$. It implies the existence and continuity of $\varphi$ but not that it is strictly increasing.

In order to prove the above theorems, we make use of the following five lemmata:

Lemma 4.4. Let $G(f) \subset K$ be an iteration group of $f$. Then $f_{\alpha}^{\prime}(0)=k^{\alpha}$ for every $f_{\alpha} \in G(f)$.

Proof. By definition, $f_{\alpha}^{\prime}(0)$ satisfies (4), and therefore $h(\alpha)=\log f_{\alpha}^{\prime}(0)$ is defined for every $\alpha$, and because it is monotone it is bounded in every finite interval. From (1) it follows that

i.e. that

$$
f_{\alpha+\beta}^{\prime}(0)=f_{\alpha}^{\prime}(0) \cdot f_{\beta}^{\prime}(0)
$$

It is a well-known fact (see e.g. [1]) that every solution of this equation, bounded in a finite interval, is of the form $h(\alpha)=c \alpha$, where $c$ is constant. This gives the lemma.

Lemma 4.5. For any $f$ with $k<1, \varphi$ has the following properties.

(a) Let $x>0$. Then for every integer $m,\left(f_{m}(x), x\right)$ belongs to $D_{\varphi}$, and we have

$$
\varphi\left(f_{m}(x), x\right)=k^{m} \text {. }
$$

(b) For $y$ fixed, $\varphi(x, y)$ is an increasing function of $x ;(x, y) \in D_{\varphi}$.

For $x$ fixed, $\varphi(x, y)$ is a decreasing function of $y ;(x, y) \in D_{\varphi}$.

(c) $\varphi(x, y)>0 ;(x, y) \in D_{\varphi}$.

(d) If any two of $(x, z),(x, y)$ and $)(y, z)$ belong to $D_{\varphi}$, then the third also belongs to $D_{\varphi}$, and

$$
\varphi(x, z)=\varphi(x, y) \cdot \varphi(y, z)
$$

(e) $\varphi(+0, y)=0, \varphi(\infty, y)=\infty$, $\varphi(x,+0)=\infty, \varphi(x, \infty)=0$.

Proof.

$$
\varphi\left(f_{m}(x), x\right)=\lim _{n \rightarrow \infty} \varphi_{n}\left(f_{m}(x), x\right)=\lim _{n \rightarrow \infty} \frac{f_{n} f_{m}(x)}{f_{n}(x)}=\lim _{n \rightarrow \infty} \frac{f_{m} f_{n}(x)}{f_{n}(x)}=f_{m}^{\prime}(0)=k^{m},
$$

which proves $(a) . b$ ) follows immediately from the fact that $\varphi_{n}$, for every $n$, is

I Convexity and concavity conditions in connection with iteration are also treated in Fort [3] and in M. Kuczma, On the Schröder equation, to appear in Rozprawy Matematyczne. 


\section{A. LUNDBERG, Iterated functions with asymptotic conditions at a fixpoint}

(strictly) increasing with respect to $x$ and decreasing with respect to $y$. To prove $(c)$, we choose an arbitrary point $(x, y)$ in $D_{\varphi}$ and an integer $m$ such that $f_{m}(y)<x$ (the latter is possible because of Lemma 3.1). Then $\varphi(x, y)=\varphi\left(f_{m}(x), f_{m}(y)\right)$ $\geqslant \varphi\left(f_{m}(x), x\right)=k^{m}>0$. (d) follows from the fact that the equality (9) holds if we replace $\varphi$ by an arbitrary $\varphi_{n}$. As for $(e)$, we prove only that $\varphi(+0, y)=0$, the other equalities being quite analogous. Keeping $y$ fixed, for every $\varepsilon>0$ there is an $m$ such that $k^{m}<\varepsilon$. Then for all $x<f_{m}(y)$ with $(x, y) \in D_{\varphi}$, we have $\varphi(x, y)=\varphi\left(f_{m}(x), f_{m}(y)\right) \leqslant \varphi\left(f_{m}(x), x\right)=k^{m}<\varepsilon$.

Lemma 4.6. Let a be a given number $>0$. If $\varphi(x, a)$ exists for all $x>0$, then $D_{\varphi}=Q$. If $\varphi(x, a)$ is continuous for $x>0$, then $\varphi(x, y)$ is continuous in $Q$.

Proof. The lemma follows immediately from $4.5(d)$ if we observe that $(x, a) \in D_{\varphi}$ implies $(a, x) \in D_{\varphi}$.

Lemma 4.7. If $\varphi$ is continuous in $Q$, then $\varphi_{n}$ converges uniformly on every compact subset of $Q$.

Proof. The statement is an easy consequence of the known fact that if a sequence of functions of one real variable, which are continuous and monotonic in an interval, converges to a continuous limit function, then the convergence is uniform on every compact subset. tions:

Finally, we have to use the following well-known properties of convex func-

Lemma 4.8. (a) Let $p$ and $q$ be increasing, convex [concave] functions. Then the composition $p q$ is convex [concave] whenever it is defined.

(b) Let $\left\{p_{n}\right\}$ be a sequence of functions, convex [concave] on an interval $I$, and assume that the sequence has a limit function $p$. Then $p$ also is convex [concave] on $I$.

(c) A function convex [concave] on an interval $I$ is continuous in the interior of $I$.

Proof of Theorem 4.2. Suppose $\left\{f_{\alpha}\right\}=G(f) \subset K$, and let $a>0$ be fixed. From Lemma 3.2 it follows that for each $x>0$ there is one and only one $\alpha$ such that $f_{\alpha}(a)=x$. We then have

$$
\varphi(x, a)=\varphi\left(f_{\alpha}(a), a\right)=\lim _{n \rightarrow \infty} \frac{f_{n} f_{\alpha}(a)}{f_{n}(a)}=\lim _{n \rightarrow \infty} \frac{f_{\alpha} f_{n}(a)}{f_{n}(a)}=f_{\alpha}^{\prime}(0)=k^{\alpha},
$$

the last equality by Lemma 4.4. Because both $f_{\alpha}(a)$ and $k^{\alpha}$ are strictly decreasing, continuous functions of $\alpha$, it follows that $\varphi(x, a)$ is a c.s.i. function of $x$.

To prove (6), we first keep in mind that in the proof above, a could be chosen quite arbitrarily. Replacing $a$ by $x$ we get $\varphi\left(f_{\alpha}(x), x\right)=k^{\alpha}$. On the other hand, by Lemma 4.5, (c) and $(d)$, we obtain

$$
\varphi\left(f_{x}(x), x\right)=\frac{\varphi\left(f_{\alpha}(x), a\right)}{\varphi(x, a)} .
$$

These two facts together give (6). 
For fixed $\alpha, f_{\alpha}$ is uniquely determined by (6) because $\varphi(x)$ is invertible, $\varphi$ is uniquely determined by $f$ and $a$, and changing $a$ is the same as multiplying (6) by a positive constant. Therefore $G(f)$ is unique.

Now suppose that for some number $a>0, \varphi(x, a)=\varphi(x)$ is defined and c.s.i. for $x>0$. Define $\varphi(0)=0$. Thus $\varphi \in S$ because of Lemma $4.5(e)$. We then define $G(f)=\left\{f_{\alpha}\right\}$ by means of $(6)$, i.e. $f_{\alpha}(x)=\varphi_{-1}\left(k^{\alpha} \cdot \varphi(x)\right)$. This makes $G(f)$ a continuous iteration group because of Lemma 3.4, and it is an iteration group of $f$ because of (13) below.

It remains to prove that $f_{\alpha}$ is differentiable at 0 with derivative $k^{\alpha}$. Let $\left\{x_{j}\right\}$ be an arbitrary sequence with $x_{j}>0$ and $\lim _{j \rightarrow \infty} x_{j}=0$. The thing to show is that for any $\alpha$

$$
\lim _{j \rightarrow \infty} \frac{f_{\alpha}\left(x_{j}\right)}{x_{j}}=k^{\alpha}
$$

Let $c$ be a fixed number $>0$. Then for each $j$ there exists an integer $n_{j}$ and a number $t_{j} \in[f(c), c]$ such that $x_{j}=f_{n_{j}}\left(t_{j}\right)$. We observe that $n_{j} \rightarrow \infty$ when $j \rightarrow \infty$. $\varphi(x, a)$ is defined and continuous for $x>0$, hence $\varphi(x, y)$ is defined and continuous in $Q$ because of Lemma 4.6, Therefore by Lemma $4.7, \varphi_{n}(x, y)$ converges uniformly to $\varphi(x, y)$ on the closed rectangle

$$
R=\left\{(x, y) \mid f_{1+\alpha}(c) \leqslant x \leqslant f_{\alpha}(c), \quad f(c) \leqslant y \leqslant c\right\},
$$

i.e. for a given $\varepsilon>0$ there exists $N(\varepsilon)$ such that $\left|\varphi(x, y)-\varphi_{n}(x, y)\right|<\varepsilon$ for every $n>N(\varepsilon)$ and $(x, y) \in R$. Now by definition of $\varphi_{n}$, we have

$$
\frac{f_{\alpha}\left(x_{j}\right)}{x_{j}}=\frac{f_{\alpha} f_{n_{j}}\left(t_{j}\right)}{f_{n_{j}}\left(t_{j}\right)}=\frac{f_{n_{j}} f_{\alpha}\left(t_{j}\right)}{f_{n_{j}}\left(t_{j}\right)}=\varphi_{n_{j}}\left(f_{\alpha}\left(t_{j}\right), t_{j}\right)
$$

Because $\left(f_{\alpha}\left(t_{j}\right), t_{j}\right) \in R$, there is an integer $J$ such that for $j>J$

$$
\left|\varphi\left(f_{\alpha}\left(t_{j}\right), t_{j}\right)-\varphi_{n_{j}}\left(f_{\alpha}\left(t_{j}\right), t_{j}\right)\right|<\varepsilon .
$$

We have only to choose $J$ such that $j>J$ implies $n_{j}>N(\varepsilon)$. (11) and (12) together with the fact that $\varphi\left(f_{\alpha}\left(t_{j}\right), t_{j}\right)=$ const. $=k^{\alpha}$. imply $(10)$, which completes the proof.

Proof of Theorem 4.3. We shall prove that the assumptions imply that for some number $a>0, \varphi(x, a)$ exists and is c.s.i. for $x>0$. But it is enough to prove it for $0<x<d$. Suppose that it is true for $0<x<d$, then from (9) it follows (cf. Lemma 4.6) that $\varphi(x, y)$ exists for all $0<x<d, 0<y<d$. But $\varphi\left(f_{m}(x), f_{m}(y)\right)=\varphi(x, y)$ for every integer $m$, and $m$ can be chosen such that $0<f_{m}(x)<d$ and $0<f_{m}(y)<d$ because of Lemma 3.1. Hence we obtain that $\varphi(x, y)$ exists in the whole of $Q$. By means of (8) and (9) we obtain that

$$
\varphi\left(f_{m}(x), a\right)=k^{m} \cdot \varphi(x, a)
$$

for every integer $m$. This gives that $\varphi$ is c.s.i. with respect to $x$, for all $x>0$. 
4.3. (a): It has already been proved by Szekeres [5] that $\varphi(x, a)$ (by Szekeres called $\chi$ ) is strictly increasing and continuous for $0<x<d$. Hence $f \in K$.

We may point out that from (7) it follows that $\varphi(x)$ is differentiable at zero, and that $0<\varphi^{\prime}(0)<\infty$ (proved by Szekeres). Therefore, in this case Theorem 4.2 is superfluous for proving that $f_{\alpha}^{\prime}(0)$ exists.

$4.3(b)$ : Assume $f(x) / x$ is increasing for $0<x<d$. Suppose first $x \leqslant a$. For sufficiently large $n, f_{n}(x)<d$ and $f_{n}(a)<d$, which gives

$$
\begin{gathered}
\frac{f f_{n}(x)}{f_{n}(x)} \leqslant \frac{f f_{n}(a)}{f_{n}(a)}, \\
\text { i.e, } \quad \varphi_{n+1}(x, a) \leqslant \varphi_{n}(x, a) .
\end{gathered}
$$

If $x \geqslant a$, we see in the same way that $\varphi_{n}(x, a)$, for sufficiently large $n$, is increasing with respect to $n$. Further, $\varphi_{n}(x, a)$ is bounded; $\varphi_{n}(x, a)>0$ and, if we choose an integer $m$ such that $f_{m}(a)>x$, we obtain $\varphi_{n}(x, a)<\varphi_{n}\left(f_{m}(a), a\right) \rightarrow k^{m}$ because of Lemma $4.5(a)$. Therefore $\varphi(x, a)$ exists for all $x>0$.

Now we show that for $x_{1}<x_{2}<d$ we have $\varphi\left(x_{1}, a\right)<\varphi\left(x_{2}, a\right)$. Because of (9), it is enough to suppose that $x_{1}<a<x_{2}$. Since $\varphi_{n}(x, a)$ is strictly increasing with respect to $x$, we have

$$
\varphi_{n}\left(x_{1}, a\right)<\varphi_{n}(a, a)<\varphi_{n}\left(x_{2}, a\right) .
$$

The proposition follows from the fact that $\varphi_{n}\left(x_{1}, a\right)$ is decreasing and $\varphi_{n}\left(x_{2}, a\right)$ is increasing with respect to $n$.

It remains to show that $\varphi(x)$ is continuous in $(0, d)$. We prove that $\varphi(x)$ is continuous in every interval $(b, c), 0<b<c<d$. In this interval we have: $\varphi(x, b)$ $[\varphi(x, c)]$ is the limit of an increasing [a decreasing] sequence of continuous functions, hence lower [upper] semi-continuous.

But because of $(9)$,

$$
\varphi(x, a)=\frac{\varphi(x, b)}{\varphi(a, b)}=\frac{\varphi(x, c)}{\varphi(a, c)},
$$

i.e. $\varphi(x, a)$ is both upper and lower semi-continuous, hence continuous.

$4.3(c)$ : The existence of $\varphi$ is proved as in $4.3(b)$, the only difference being that $\varphi_{n}(x, a)$ is, with respect to $n$, increasing for $x \leqslant a$ and decreasing for $x \geqslant a$. By Lemma $4.8(a)$ and $(b), \varphi$ is concave in $(0, d)$ and therefore, by Lemma $4.8(c)$, continuous in $(0, d)$. We know that $\varphi(x)$ is increasing, and we have only to show that it is strictly increasing. Let $f\left(x_{2}\right)<x_{1}<x_{2}<d$, and suppose $\varphi\left(x_{1}\right)=$ $\varphi\left(x_{2}\right)$. Then (13) gives that $\varphi f\left(x_{1}\right)=k \cdot \varphi\left(x_{1}\right)=k \cdot \varphi\left(x_{2}\right)=\varphi f\left(x_{2}\right)<\varphi\left(x_{1}\right)$, which contradicts the fact that $\varphi$ is concave. This completes the proof of Theorem 4.3.

\section{Transformation of the class $K$}

The theorems of Section 4 can be applied to many functions not belonging to $K$, because every iteration group can be transformed into an iteration group included in $K$. 
Definition 5.1. Suppose $F \in S$; the function $f \in S_{0}$ is said to belong to the class $K\left(F^{\prime}\right)$ if, and only if, $F f F_{-1} \in K$.

If $g=F f F_{-1} \in K$, there is a unique iteration group $\left\{g_{\alpha}\right\} \subset K$ and, by Lemma 3.4, the set $G(f)=\left\{f_{\alpha} \mid f_{\alpha}=F_{-1} g_{\alpha} F\right\}$ is an iteration group of $f$. Supposing $g_{\alpha}^{\prime}(0)=k^{\alpha}$, $0<k \neq 1$, we have

$$
\lim _{x \rightarrow 0} \frac{F f_{\alpha}(x)}{F(x)}=k^{\alpha} .
$$

Conversely, if $G(f)$ is an iteration group of $f$ satisfying (14), the set $\left\{g_{\alpha} \mid g_{\alpha}=\right.$ $\left.F f_{\alpha} F_{-1}\right\}$ is an iteration group in $K$ with $g_{\alpha}^{\prime}(0)=k^{\alpha}$. Thus $K\left(F^{\prime}\right)$ is the union of all the continuous iteration groups the elements of which satisfy (14) for some positive constant $k \neq 1$.

A class $K\left(F^{\prime}\right)$ may be determined in different ways. For instance, two different functions $F_{1}$ and $F_{2}$ may determine the same class:

Lemma 5.2. Assume that there are two positive constants $b$ and $c$ such that $\lim _{x \rightarrow 0} F_{1}(x) \cdot\left(F_{2}(x)\right)^{-b}=c$. Then $K\left(F_{1}\right)=K\left(F_{2}\right)$.

Proof. It is enough to prove that $f \in K\left(F_{1}\right)$ implies $f \in K\left(F_{2}\right)$. Suppose

$$
\lim _{x \rightarrow 0} \frac{F_{1} f_{\alpha}(x)}{F_{1}(x)}=k^{\alpha} \text {. }
$$

This gives immediately

$$
\lim _{x \rightarrow 0} \frac{F_{2} f_{\alpha}(x)}{F_{2}(x)}=k^{\alpha / b},
$$

i.e. $f \in K\left(F_{2}\right)$.

Lemma 5.3. The class $K(F)$ is the same for all Schröder functions $F$ of a given iteration group.

The proof follows immediately from Lemmata 3.5 and 5.2.

A class $K(F)$ is also determined by a given iteration group, which is shown below. For this and for later purposes, we introduce the following notations. Let $G(g)=\left\{g_{\alpha}\right\}$ be a given iteration group and let $h \in S_{0}$ be a given function. Then by Lemma 3.2 , for every $x>0$ there is one and only one number $\lambda=\lambda(h, x)$ such that

$$
g_{\lambda}(x)=h(x)
$$

If $h=f_{\alpha} \in G(f)$, where $G(f)$ is a given iteration group, then for brevity we write $\lambda(\alpha, x)$ instead of $\lambda\left(f_{\alpha}, x\right)$. The function $\lambda(\alpha, x)$ satisfies the relation

$$
\lambda(\alpha+\beta, x)=\lambda\left(\alpha, f_{\beta}(x)\right)+\lambda(\beta, x)
$$

for if $\lambda_{1}=\lambda\left(\alpha, f_{\beta}(x)\right)$ and $\lambda_{2}=\lambda(\beta, x)$, then $g_{\lambda_{1}+\lambda_{2}}(x)=g_{\lambda_{1}} g_{\lambda_{z}}(x)=g_{\lambda_{1}} f_{\beta}(x)=f_{\alpha} f_{\beta}(x)=$ $=f_{\alpha+\beta}(x)$.

For fixed $x$, the mapping $\alpha \rightarrow \lambda(\alpha, x)$ is obviously strictly monotone. 


\section{A. LUNDBERG, Iterated functions with asymptotic conditions at a fixpoint}

Lemma 5.4. Assume $G(g) \subset K\left(F^{\prime}\right)$. Then $G(f) \subset K(F)$ if, and only if, for every $\alpha$ $\lambda(\alpha, 0)=\lim _{n \rightarrow 0} \lambda(\alpha, x)$ exists and $\lambda(\alpha, 0) \neq 0$ for $\alpha \neq 0$. In this case, there is $a$ number $c \neq 0$ such that

$$
\lambda(\alpha, 0)=c \alpha
$$

Proof. It is no restriction to suppose that $g>e$. Then by assumption there is a number $k>1$ such that $\lim _{x \rightarrow 0}\left(F g_{\alpha}(x) / F(x)\right)=k^{\alpha}$. Now first suppose that $\lambda(\alpha, 0)$ exists and is non-zero for $\alpha \neq 0$. Since $\lambda(\alpha, 0)$ is non-decreasing, (17) follows immediately from (16) (cf. Lemma 4.4). Obviously $c \neq 0$. If $\varepsilon>0$ we then have

$$
\varlimsup_{x \rightarrow 0} \frac{F f_{\alpha}(x)}{F(x)}=\varlimsup_{x \rightarrow 0} \frac{F g_{\lambda}(x)}{F(x)} \leqslant \lim _{x \rightarrow 0} \frac{F g_{c \alpha+\varepsilon}(x)}{F(x)}=k^{c \alpha+\varepsilon},
$$

and in the same way $\lim _{x \rightarrow 0}\left(F f_{\alpha}(x) / F(x)\right) \geqslant k^{c \alpha-\varepsilon}$, which gives $\lim _{x \rightarrow 0}\left(F f_{\alpha}(x) /\right.$ $F(x))=k^{c \alpha}$, since $\varepsilon>0$ is arbitrary.

Conversely, suppose for some $c \neq 0$ that $\lim _{x \rightarrow 0}\left(F f_{\alpha}(x) / F(x)\right)=k^{c \alpha}$. If $\mu=$ $\overline{\lim }_{x \rightarrow 0} \lambda(\alpha, x)$ and $\nu=\lim _{x \rightarrow 0} \lambda(\alpha, x)$, for $\varepsilon>0$ we easily obtain

$$
k^{\mu-\varepsilon} \leqslant k^{c \alpha} \leqslant k^{\nu+\varepsilon},
$$

which gives $\mu=\nu=c \alpha$, and the lemma is proved.

Theorem 5.5. $K\left(F_{1}\right)=K\left(F_{2}\right)$ if, and only if, they have a common iteration group, i.e. an iteration group belonging to a class $K(F)$ determines the class uniquely.

Proof. Because of symmetry it is sufficient to prove that $f \in K\left(F_{1}\right)$ implies $f \in K\left(F_{2}\right)$. Let $G(g)$ be the common iteration group, and let $\left\{f_{\alpha}\right\} \subset K\left(F_{1}\right)$ be the uniquely determined iteration group of $f$. Because of Lemma 5.4, there is a number $c \neq 0$ such that $\lim _{x \rightarrow 0} \lambda(\alpha, x)=c \alpha$. From

$$
\lim _{x \rightarrow 0} \frac{F_{2} g_{\alpha}(x)}{F_{2}(x)}=k^{\alpha}
$$

we obtain

$$
\lim _{x \rightarrow 0} \frac{F_{2} f_{\alpha}(x)}{F_{2}(x)}=k^{c \alpha},
$$

i.e. $\left\{f_{\alpha}\right\} \subset K\left(F_{2}\right)$.

\section{Asymptotic comparison of iteration groups}

With somewhat different notations, we quote the following three theorems from Michel's work [4]. The theorems have the same numbers here as in Michel's paper.

Theorem 6.1. Assume that $q>1$ and $\lim _{x \rightarrow 0}(f(x) / x)=q$. Then there exists at most one iteration group $G(f)$ such that for every $f_{\alpha} \in G(f)$, 


$$
\lim _{x \rightarrow 0} \frac{f_{\alpha}(x)}{x}=q^{\alpha}
$$

Theorem 6.2. Assume that $q \neq 0$ and

$$
\lim _{x \rightarrow 0} \frac{f(x)-x}{x^{1+\delta}}=q
$$

where $\delta>0$. Then there exists at most one iteration group $G(f)$ such that for every $f_{\alpha} \in G(f)$,

$$
\lim _{x \rightarrow 0} \frac{f_{\alpha}(x)-x}{x^{1+\delta}}=\alpha q
$$

Theorem 6.3. Assume that $q>0$ and $\lim _{x \rightarrow 0}\left(f(x) / x^{u}\right)=q$, where $0<u<1$. Then there exists at most one iteration group $G(f)$ such that for every $f_{\alpha} \in G(f)$,

$$
\lim _{x \rightarrow 0} \frac{f_{\alpha}(x)}{x^{u^{\alpha}}}=q^{\left(u^{\alpha}-1\right) /(u-1)} .
$$

Theorem 6.1 follows from Theorem 4.2. We show below that 6.2 and 6.3 also follow from 4.2, if we apply the results of Section 5 .

The theorems quoted exemplify uniqueness conditions for $G(f)$ for some types of asymptotic behaviour of $f$ at zero. Michel brings up the question of whether for any given $f$ we may find asymptotic conditions for $f_{\alpha}$ at zero which make the group $G(f)=\left\{f_{\alpha}\right\}$ unique. The purpose of the following investigation is to elucidate this question. We try to frame the question more precisely, even if at the same time we lose some aspects of the question.

In the following, we shall always let $G$ be a given iteration group of a function $g$. Let $\varrho$ be a. relation defined for the group $G$ and arbitrary functions $h \in S_{0}$. By $\varrho(G, h)$ we mean that the relation $\varrho$ holds for $G$ and $h$. If $\varrho(G, h)$ for every function $h$ in a set $M$, we write $\varrho(G, M)$.

If $\varrho(G, f)$ for a given function $f$, the following questions arise:

(1) Is there any iteration group $G(f)$ such that $\varrho(G, G(f))$ ?

(2) If the answer is yes, is $G(f)$ unique?

We now define some relations $\varrho$ to be investigated. Let $\lambda$ be the function defined by (15), where $\left\{g_{\alpha}\right\}=G$.

Definition 6.4. $\varrho_{j}(G, h), j=1,2,3,4$, is defined to be true for $h=e$. For $h \neq e$ we define:

$\varrho_{1}(G, h)$ to mean that $\lambda(h, 0)=\lim _{x \rightarrow 0} \lambda(h, x)$ exists and is different from zero;

$\varrho_{2}(G . h)$ to mean that there exists $\alpha \neq 0$ such that for every $\beta \neq 0$,

$$
\lim _{x \rightarrow 0} \frac{h(x)-g_{\alpha}(x)}{g_{\alpha+\beta}(x)-g_{\alpha}(x)}=0 ;
$$




\section{A. LUNDBERG, Iterated functions with asymptotic conditions at a fixpoint}

$\varrho_{3}(G, h)$ to mean that there exists $\alpha \neq 0$ such that $\lim _{x \rightarrow 0}\left(h(x) / g_{\alpha}(x)\right)=1$;

$\varrho_{4}(G, h)$ to mean that there exists $\alpha \neq 0$ such that $\log \left(h(x) / g_{\alpha}(x)\right)$ is bounded for $0<x<d<\infty$.

For $\varrho_{1}$ we have the following.

Theorem 6.5. Let $F$ be a Schröder function of $G$. Then $G(f) \subset K(F)$ if and only if $\varrho_{1}(G, G(f))$.

Proof. Obviously $\mathrm{G} \subset K(F)$. Therefore the theorem follows immediately fom Lemma 5.4.

This theorem, together with Theorems 4.2 and 5.5, gives a complete answer to Questions 1 and 2 for the case $\varrho=\varrho_{1}$. In fact, let $f$ be a function in $S_{0}$ with $\varrho_{1}(G, f)$. By means of Theorem 4.2 we may determine whether or not $f \in K(F)$ (i.e. $\left.F f F_{-1} \in K\right)$. This gives the answer to Question 1 , because of Theorem 6.5. If $f \in K\left(F^{\prime}\right)$, the uniqueness of $G(f)=\left\{f_{\alpha}\right\}$ satisfying $\varrho_{1}(G, G(f))$, follows from Theorem 5.5.

For $\varrho=\varrho_{j}, j=2,3,4$, we have the following

\section{Theorem 6.6.}

(a) $\varrho_{2}(G, h)$ implies $\varrho_{1}(G, h)$.

(b) If $\lim _{x \rightarrow 0}\left|g_{\alpha}(x) / x-1\right|>0$ for $\alpha \neq 0$, then $\varrho_{3}(G, h)$ implies $\varrho_{1}(G, h)$.

(c) If $\overline{g_{\alpha}(x)} / x \rightarrow 0$ or $\infty$ when $x \rightarrow 0$, for every $\alpha \neq 0$, then $\varrho_{4}(G, h)$ implies $\varrho_{1}(G, h)$.

Proof. It is enough to prove the theorem for $g>e$. In the proofs of $(a),(b)$ and $(c)$ below, we let $\alpha$ denote the numbers in the definitions of $\varrho_{2}, \varrho_{3}$ and $\varrho_{4}$ respectively.

(a) Suppose $\varrho_{2}(G, h)$. We first show that $\varlimsup_{\lim _{x \rightarrow 0}} \lambda(h, x) \leqslant \alpha$. If this were false, there would exist an $\varepsilon>0$ and a sequence $\left\{x_{j}\right\}$, where $x_{j} \rightarrow 0$ when $j \rightarrow \infty$, such that $\lambda\left(h, x_{j}\right)>\alpha+\varepsilon$ for every $j$. Then, because of Lemma 3.2, we would get

$$
\frac{h\left(x_{j}\right)-g_{\alpha}\left(x_{j}\right)}{g_{\alpha+\varepsilon}\left(x_{j}\right)-g_{\alpha}\left(x_{j}\right)}>\frac{g_{\alpha+\varepsilon}\left(x_{j}\right)-g_{\alpha}\left(x_{j}\right)}{g_{\alpha+\varepsilon}\left(x_{j}\right)-g_{\alpha}\left(x_{j}\right)}=1,
$$

in contradiction to $\varrho_{2}(G, h)$. In the same way, we prove $\underline{\lim }_{x \rightarrow 0} \lambda(h, x) \geqslant \alpha$, which gives $\varrho_{1}(G, h)$.

(b) We easily obtain that if $\lim _{x \rightarrow 0}\left|g_{\alpha}(x) / x-1\right|>0$, then $\varrho_{3}(G, h) \Rightarrow \varrho_{2}(G, h)$, i.e. the proposition follows immediately from $(a)$.

(c) If $g>e$, then the assumption is that $g_{\alpha}(x) / x$ tends to $\infty$ for $\alpha>0$ and to 0 for $\alpha<0$, when $x$ tends to 0 . Now suppose $\overline{\lim }_{x \rightarrow 0} \lambda(h, x)>\alpha$, i.e. suppose there is a number $\varepsilon>0$ and a sequence $\left\{x_{j}\right\}$ with $\lim _{j \rightarrow \infty} x_{j}=0$, such that $\lambda\left(h, x_{j}\right)>\alpha+\varepsilon$. Then

$$
\frac{h\left(x_{j}\right)}{g_{\alpha}\left(x_{j}\right)}>\frac{g_{\alpha+\varepsilon}\left(x_{j}\right)}{g_{\alpha}\left(x_{j}\right)}=\frac{g_{\varepsilon} g_{\alpha}\left(x_{j}\right)}{g_{\alpha}\left(x_{j}\right)} \rightarrow \infty,
$$

when $j \rightarrow \infty$. This contradicts $\varrho_{4}(G, h)$, i.e. $\varrho_{4}(G, h)$ implies $\varlimsup_{\lim _{x \rightarrow 0}} \lambda(h, x) \leqslant \alpha$. In 
the same way we show that $\varrho_{4}(G, h)$ implies $\lim _{x \rightarrow 0} \lambda(h, x) \geqslant \alpha$. This gives that $\varrho_{4}(G, h)$ implies $\varrho_{1}(G, h)$, which was to be proved.

The Theorems 6.2 and 6.3 can be obtained as applications of Theorem 6.6. Applying $6.6(a)$ with

$$
g_{\alpha}(x)=\frac{x}{\left(1+\alpha x^{\delta}\right)^{1 / \delta}}
$$

for sufficiently small $x$, and $6.6(c)$ with $g_{\alpha}(x)=x^{u^{\alpha}}$ for sufficiently small $x$ we obtain Theorems 6.2 and 6.3 respectively. The existence of groups with the mentioned properties follows from the investigation in Section 5 of Michel's work [4].

Of course, it is desirable to make a uniqueness condition of the type $\varrho(G, G(f))$ as weak as possible. In $(a),(b)$ and $(c)$ of Theorem 6.7 , the conditions $\varrho_{j}(G, G(f)), j=2,3,4$, are stronger than $\varrho_{1}(G, G(f))$. Therefore, the only interest of Theorem 6.7 lies in the fact that $\varrho_{j}(G, h), j=2,3,4$, expresses the behaviour of $h$ more concretely than $\varrho_{1}(G, h)$. Whether there is any $G$ with the property that there exists an $f$ with unique $G(f)$, fulfilling $\varrho_{j}(G, G(f)), j=3$ or 4 but not 1, will not be determined here.

We conclude this paper by touching upon the problem of weakening $\varrho_{1}(G, G(f))$. Maybe there are different natural ways of weakening this condition, but we consider only the following: from Lemma 5.4 we know that $\varrho_{1}(G, G(f))$ is equivalent to the existence of a $c \neq 0$ such that $\lim _{x \rightarrow 0}|\lambda(\alpha, x)-c \alpha|=0$. By Lemma 3.3 it is no restriction to assume $c=1$. Let $\theta$ be a non-negative function, and define.

$$
\varrho_{\theta}(G, G(f)) \Leftrightarrow \varlimsup_{x \rightarrow 0}|\lambda(\alpha, x)-\alpha| \leqslant \theta(\alpha)
$$

If $\theta(\alpha)>0$ for some $\alpha$, then $\varrho_{\theta}(G, G(f))$ is formally weaker than $\varrho_{1}(G, G(f))$, but the following example shows that there are $\theta(\alpha)$, not identically 0 , such that $\varrho_{\theta}(G, G(f)) \Leftrightarrow \varrho_{1}(G, G(f)):$

$$
\text { Example 6.7. } \theta(0)=\theta^{\prime}(0)=0 .
$$

To show that if $\theta$ has this property, $\varrho_{\theta}(G, G(f)) \Rightarrow \varrho_{1}(G, G(f))$, we put

$$
l(\alpha)=\varlimsup_{x \rightarrow 0}|\lambda(\alpha, x)-\alpha|
$$

From (16) we easily obtain

$$
l(\alpha+\beta) \leqslant l(\alpha)+l(\beta)
$$

Now we fix a number $\alpha$ and put $\alpha=n \beta$, where $n$ is a positive integer. From (18) and (20) we get

$$
l(\alpha)=l(n \beta) \leqslant n l(\beta) \leqslant n \theta(\beta)=\alpha \cdot(n / \alpha) \cdot \theta(\alpha / n),
$$

which tends to 0 when $n \rightarrow \infty$, because of (19). This implies $\varrho_{1}(G, G(f))$.

The next example shows that $\theta(\alpha)$ must take arbitrarily small values to give uniqueness: 
A. LUNDBERG, Iterated functions with asymptotic conditions at a fixpoint

Example 6.8.

$$
\theta(\alpha) \geqslant \varepsilon>0 \text { for every } \alpha .
$$

In this case, we may construct an iteration group $G^{*}(g) \neq G$ such that $\varrho_{\theta}\left(G, G^{*}(g)\right)$. Let $A$ be an Abel function of $G$, and let $p$ be a non-constant function with period 1 such that $p+e$ is c.s.i. and, for every $x$

$$
|p(x)|<\varepsilon / 2 \text {. }
$$

By .Lemma 3.6, $G^{*}(g)=\left\{g_{\alpha}^{*} \mid g_{\alpha}^{*}(0)=0, g_{\alpha}^{*}(x)=B_{-1}(\alpha+B(x))\right.$ for $\left.x>0\right\}$ is an iteration group of $g$ different from $G$ if we define $B(x)=A(x)+p A(x)$. By definition of Abel functions, we have

$$
A g_{\lambda(\alpha, x)}(x)=\lambda(\alpha, x)+A(x)
$$

and by definition of $G^{*}(g)$,

$$
p A g_{\lambda(\alpha, x)}(x)+A g_{\lambda(\alpha, x)}(x)=\alpha+A(x)+p A(x),
$$

where $g_{\lambda(\alpha, x)}=g_{\alpha}^{*}(x)$. Combining (21), (22) and (23), we get

$$
|\lambda(\alpha, x)-\alpha|<|p A(x)|+\left|p A g_{\lambda_{(\alpha, x)}}(x)\right|<\varepsilon .
$$

This shows that there is at least one function $f$ (namely $f=g$ ) with more than one iteration group $G(f)$ satisfying $\varrho_{\theta}(G, G(f))$.

These two examples can be considerably improved, and the following question arises: Is there any function $\theta(\alpha)$ such that $\varrho_{\theta}(G, G(f))$ is a uniqueness condition for $G(f)$, strictly weaker than $\varrho_{1}(G, G(f))$ ? This question, however, will not be answered here.

\section{A C K N O W L E D G EM E N T}

I want to thank my teacher, Professor Otto Frostman, for his valuable assistance and fruitful criticism during my work.

\section{REFEREN CES}

1. Aczíl, J., Vorlesungen über Funktionalgleichungen und ihre Anwendungen. Birkhäuser Verlag, Basel und Stuttgart, 1961.

2. Aczér, J., Kalmár, L., and Mikusiński, J. G., Sur l'équation de translation. Studia Math. 12 (1951), $112-116$.

3. Font, M. K. Jr., The embedding of homeomorphisms in flows. Proc. Amer. Math. Soc. 6 (1955), 960-967.

4. Michel, H., Untersuchungen über stetige, monotone Iterationsgruppen reeller Funktionen ohne Differenzierbarkeitsvoraussetzungen. Publ. Math. Debrecen 9 (1962), 13-46.

5. Szekeres, G., Regular iteration of real and complex functions. Acta Math. 100 (1958), $203-258$.

6. Töpfer, H., Komplexe Iterationsindizes ganzer und rationaler Funktionen. Math. Ann. $121(1949), 191-222$.

7. WAlker, A. G., Commutative functions (I). Quart. J. Math. (Oxford) 17 (1945), 65-82. 